\title{
Results with diffractive parton distributions for the HERA, the Tevatron and the LHC
}

\section{Agnieszka Łuszczak*}

Institute of Nuclear Physics Polish Academy of Sciences, Cracow, Poland

E-mail: agnieszka.luszczakeifj.edu.pl

We present theoretical studies of diffractive parton distributions, which we determined from QCD fits to $\mathrm{H} 1$ and ZEUS data from $e p$ collisions at the HERA, and used for $p \bar{p}$ collisions at the Tevatron and $p p$ collisions at the LHC.

XXth Hadron Collider Physics Symposium

November 16 - 20, 2009

Evian, France

*Speaker.

${ }^{\dagger}$ This work is partially supported by the grant of MNiSW N202 249235. 


\section{Diffractive parton distributions from HERA}

The determination of diffractive parton distributions, was performed in the framework of the QCD fits to the diffractive data from HERA. In this analysis, we included a higher twist-4 contribution, taken form the dipole models, which dominates for small diffractive masses, $M^{2} \ll Q^{2}$. We proved that this contribution is important for the determination of the diffractive gluon distribution. It also has a big impact on the diffractive longitudinal structure function, $F_{L}^{D}$, for large values of the variable $\beta$ (small diffractive masses) [1]. The latter result can be compared with measurements when the results from the low energy runs at HERA will be available.

We compared two sets of diffractive parton distributions, from the DGLAP fits and from the dipole model approach analyzes. We found significant difference between them, especially for the diffractive gluon distributions. Based on these results, we made predictions for the diffractive charm production using the boson-gluon fusion process, $\gamma^{*} g \rightarrow c \bar{c}$, with the diffractive gluon distribution taken from the two discussed analyzes. We found reasonable agreement with the first HERA data. We also confirmed the known result that the pure $c \bar{c}$ diffractive production is strongly suppressed due to kinematic limits. The above results for HERA are presented in details in $[2,3]$.

\section{Diffractive parton distributions from HERA to Tevatron}

The diffractive parton distributions (DPD) are not universal and cannot be applied to both lepton-hadron and hadron-hadron diffractive processes. According to [5], additional soft interactions between colliding hadrons prevent the collinear factorization universality of the DPD. This is seen in diffractive production of dijets at the Tevatron, which we discussed. We used the DPD from HERA to show the scale of the factorization breaking and discuss the role of the secondary reggeon contribution in the description of this effect. We also discussed the gap survival probability for the dijet production. To summarize, factorization does not occur between HERA and Tevatron because of the long term additional soft exchanges between spectators in the colling hadrons. Nevertheless, experimentally, factorization happens in case of CDF data themselves and also between single diffraction and double pomeron exchange which means that the soft exchanges do not depend on hard scattering.

\section{Diffractive parton distributions from HERA to LHC}

We describe diffractive hadroproduction of $W / Z$ bosons and give predictions for the single diffractive boson production cross sections at the LHC. We discuss in detail the $W^{ \pm}$asymmetry in $p \mathbb{P}$ collisions showing that this quantity is a good observable to test the concept of the flavor symmetric pomeron parton distributions [4]. In the diffractive case, the electroweak bosons are produced in a restricted region of rapidity, with a rapidity gap without particles between the proton, which stayed intact and the diffractive system. In this process, the boson mass is a hard scale allowing for perturbative QCD interpretation as in the nondiffractive case. However, the nature of the vacuum quantum number exchange, which leads to the rapidity gap, is nonperturbative. It is usually modelled using the Regge theory notion - a Pomeron. 

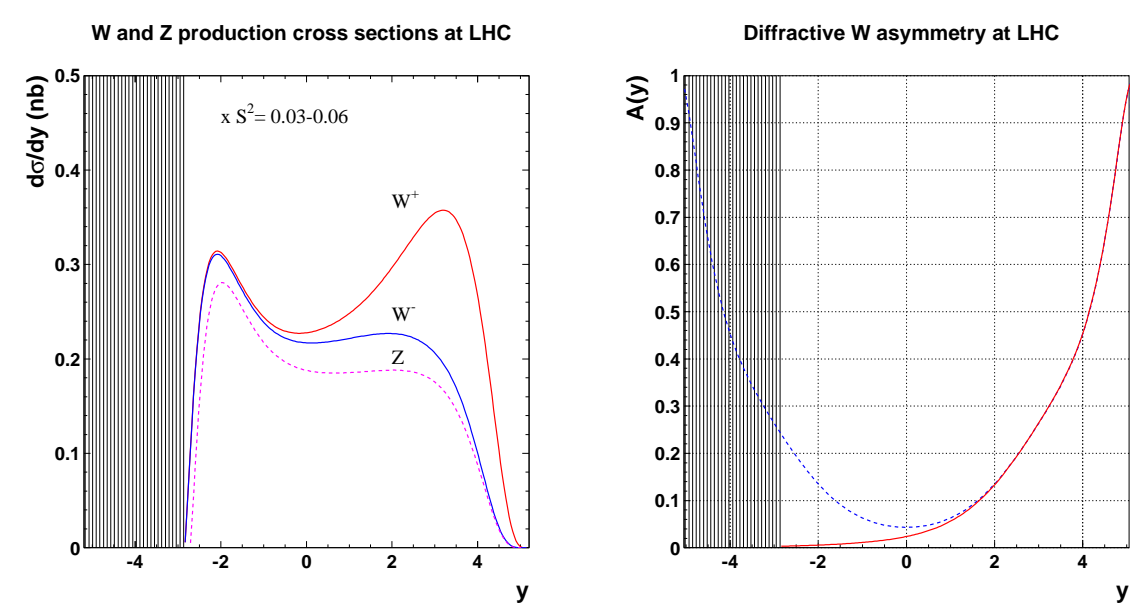

Figure 1: Left: the single diffractive $W / Z$ boson production cross sections at the LHC as functions of boson rapidity. The results have to be multiplied by the gap survival factor $S^{2}=0.09$. Right: the $W$ asymmetry in $p \mathbb{P}$ collisions (solid line), together with the asymmetry in $p p$ collisions (dashed line). The shaded areas indicate the rapidity gap $\Delta=2.3$ for $x_{\mathbb{P}}=0.1$.

In the single diffractive case, the $W$ production cross sections are related to quark distributions in the following way

$$
\begin{aligned}
\frac{d \sigma_{W^{+}}}{d y d x_{\mathbb{P}}} & \sim\left(u_{p}\left(x_{1}\right)+\bar{d}_{p}\left(x_{1}\right)\right) q_{\mathbb{P}}\left(x_{2} / x_{\mathbb{P}}\right) \\
\frac{d \sigma_{W^{-}}}{d y d x_{\mathbb{P}}} & \sim\left(d_{p}\left(x_{1}\right)+\bar{u}_{p}\left(x_{1}\right)\right) q_{\mathbb{P}}\left(x_{2} / x_{\mathbb{P}}\right) .
\end{aligned}
$$

and diffractive asymmetry:

$$
A^{D}(y)=\frac{u_{\text {val }}\left(x_{1}\right)-d_{\text {val }}\left(x_{1}\right)}{u_{\text {val }}\left(x_{1}\right)+d_{\text {val }}\left(x_{1}\right)+2\left(u_{\text {sea }}\left(x_{1}\right)+d_{\text {sea }}\left(x_{1}\right)\right)} .
$$

In Fig. 1 (left) we show the $W$ and $Z$ production cross sections with the LO MSTW08 proton parton distributions and the pomeron parton distributions from our last analysis [2]. The effect of the pomeron in the left hemisphere is clearly visible - the rapididty gap is formed and the $W^{ \pm}$ asymmetry strongly decreases. These cross sections should be multiplied by a gap survival factor, $S^{2}=0.09$ [6], which takes into account soft interactions destroying the rapidity gap.

\section{References}

[1] A. Luszczak, Acta Phys. Polon. B38, 2331 (2007).

[2] K. J. Golec-Biernat and A. Luszczak, Phys. Rev. D76, 114014 (2007), [0704.1608].

[3] K. J. Golec-Biernat and A. Luszczak, Phys. Rev. D79, 114010 (2009), [0812.3090].

[4] K. Golec-Biernat and A. Luszczak, Phys. Rev. D81, 014009 (2010), [0911.2789].

[5] J. C. Collins, L. Frankfurt and M. Strikman, Phys. Lett. B307, 161 (1993), [hep-ph/9212212].

[6] V. A. Khoze, A. D. Martin and M. G. Ryskin, Eur. Phys. J. C18, 167 (2000), [hep-ph/0007359]. 